\title{
Candidate Paths for Impairment-Aware PCE in 10-100 Gb/s Optical Networks
}

\author{
Nicola Sambo, Francesco Paolucci, Gianluca Meloni, \\ Filippo Cugini, Marco Secondini, Luca Potì, and Piero Castoldi
}

\begin{abstract}
Two impairment-aware path computation element (PCE) architectures, exploiting candidate paths, are presented. Novel PCE protocol extension is proposed to improve path computation in multi bit-rate optical networks affected by detrimental non-linear effects among $10 \mathrm{~Gb} / \mathrm{s}$ and $100 \mathrm{~Gb} / \mathrm{s}$ lightpaths. The PCE architectures have been successfully experimented on a real $10-100 \mathrm{~Gb} / \mathrm{s}$ testbed.
\end{abstract}

Index Terms-PCE, 100 Gbit/s, multi bit-rate, WSON, XPM.

\section{INTRODUCTION}

$\mathbf{T}$ HE recent advances in optical technologies and transmission techniques are rapidly enabling the evolution of wavelength switched optical networks (WSONs) to support lightpaths operating at different bit-rates and modulation formats. Lightpaths at $10 \mathrm{~Gb} / \mathrm{s}$ bit-rate with on-off keying (OOK) modulation will soon coexist in the same WSON with lightpaths operating at $100 \mathrm{~Gb} / \mathrm{s}$ with dual polarization quadrature phase shift keying (DP-QPSK). In multi bitrate WSONs, traffic dependent physical effects have to be carefully considered being more detrimental than in single bit-rate/modulation WSONs. In particular, intensity-modulated $10 \mathrm{~Gb} / \mathrm{s}$ OOK lightpaths may induce a detrimental crossphase modulation (XPM) on phase-modulated $100 \mathrm{~Gb} / \mathrm{s} \mathrm{DP}$ QPSK lightpaths [2], [3]. However, because of the walkoff between channels, the larger the spectral distance between QPSK and OOK signals, the less detrimental the effects of XPM. These effects might significantly impact the impairment-aware routing and wavelength assignment (IARWA) performance and the overall network resource utilization. In [4], the path computation element (PCE) architecture is considered to address IA-RWA. In particular, a solution called $I V$-candidates $+R W A$, or simply $I V+R W A$, is proposed. In IV+RWA, impairment validation (IV) is first performed by an IV PCE and a set of (feasible) candidate paths is identified for the subsequent (impairment-unaware) RWA, performed by an RWA PCE. The IV+RWA architecture has been proposed to address all implementation scenarios in which a single element performing combined evaluation of both IV and RWA is not considered adequate to retrieve, store and handle a large amount of both physical and networking information (e.g., because of scalability reasons). In this study, differently from

Manuscript received March 11, 2011. The associate editor coordinating the review of this letter and approving it for publication was A. Sekercioglu.

N. Sambo (e-mail: nicola.sambo@sssup.it), F. Paolucci, G. Meloni, M. Secondini, and P. Castoldi are with Scuola Superiore Sant'Anna, Pisa, Italy.

F. Cugini and L. Potì are with CNIT, Pisa, Italy.

Part of this work has been presented in [1]. This work was partially supported by the STRONGEST project.

Digital Object Identifier 10.1109/LCOMM.2011.060811.110545 other relevant experiments on impairment-aware PCEs [5][7], we consider and experimentally show the performance of the IV+RWA solution applied to the case of multi bit-rate 10$100 \mathrm{~Gb} / \mathrm{s}$ WSON. Then, differently from [1], we propose an enhanced IV+RWA PCE architecture (EIV+RWA) capable of improving the IV+RWA PCE path computation performance thanks to a novel lightweight PCE protocol (PCEP) extension.

\section{IV+RWA PCE IMPLEMENTATIONS}

\section{A. Impairment validation tools}

The two considered IV+RWA and EIV+RWA implementations exploit the same set of IV tools to estimate bit error rate (BER) of either $10 \mathrm{~Gb} / \mathrm{s}$ OOK or $100 \mathrm{~Gb} / \mathrm{s}$ DP-QPSK. The tools and related equations are detailed in [3]. The IV tool for $10 \mathrm{~Gb} / \mathrm{s}$ OOK implements an OSNR-based model accounting for amplified spontaneous emission (ASE), chromatic dispersion (CD), first-order polarization mode dispersion (PMD), and self phase modulation (SPM) through non-linear phase shift $\phi_{\mathrm{NL}}$. Effects of CD and $\phi_{\mathrm{NL}}$ are computed as penalty to the OSNR. Then, BER is derived from the final OSNR [3]. The IV tool for $100 \mathrm{~Gb} / \mathrm{s}$ DP-QPSK with coherent detection accounts for ASE, SPM, and XPM, and estimates BER through the Gaussian approximation in [3]. In the case of $100 \mathrm{~Gb} / \mathrm{s}$, $\mathrm{CD}$ and PMD are not considered since coherent detection enables an electronic post processing which compensates the effects of dispersion. XPM is particularly detrimental (thus, accounted for) on $100 \mathrm{~Gb} / \mathrm{s}$ lightpaths if induced by $10 \mathrm{~Gb} / \mathrm{s}$ lightpaths [3]. The IV tool for $100 \mathrm{~Gb} / \mathrm{s}$ considers XPM in two ways: in the worst-case scenario or with guard band $(G B)$ [3]. The worst-case scenario for $100 \mathrm{~Gb} / \mathrm{s}$ occurs when the central wavelength is occupied by the $100 \mathrm{~Gb} / \mathrm{s}$ lightpath surrounded by $10 \mathrm{~Gb} / \mathrm{s}$ lightpaths. If a $100 \mathrm{~Gb} / \mathrm{s}$ lightpath has acceptable BER in the worst-case scenario, its BER is acceptable with any traffic condition and any wavelength along the path is admitted (i.e., it can be selected since it guarantees the BER requirements). Alternatively, 10 and $100 \mathrm{~Gb} / \mathrm{s}$ lightpaths can be spectrally separated by GB, where GB is defined as the number of free wavelengths guaranteeing a negligible XPM in any path. In this case, only a subset of wavelengths along these paths is admitted, i.e. guaranteeing a spectral separation of GB among 10 and $100 \mathrm{~Gb} / \mathrm{s}$ lightpaths. The adoption of worst-case scenario or GB depends on the considered PCE architecture implementation, as described in the following.

\section{B. PCE Architecture implementations}

The first considered IV+RWA implementation follows the basic indications provided in [4]. The connection request generated by a path computation client (PCC) is handled by 


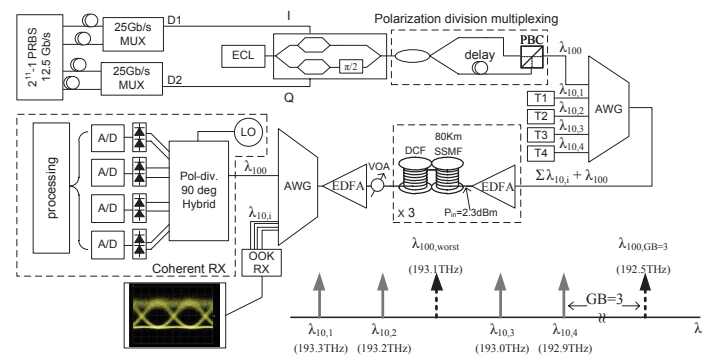

Fig. 1. Experimental testbed.

the RWA PCE which forwards the PCEP PCReq message to the IV PCE. Such message includes, besides end-points and requested bit-rate, a candidate object specifying the number $K$ of expected validated paths. The IV PCE runs impairment estimation tools on the basis of the physical information included in its traffic engineering database (TED) (e.g., provided and maintained by the network management system). IV PCE computes the set of feasible candidate paths, i.e. having acceptable BER (BER $\left.<\mathrm{BER}_{\mathrm{TH}}\right)$. Since RWA is unknown at this stage, any wavelength has to guarantee acceptable BER, i.e. any available wavelength needs to be admitted. To this purpose, XPM due to $10 \mathrm{~Gb} / \mathrm{s}$ is considered for $100 \mathrm{~Gb} / \mathrm{s}$ only in the worst-case scenario. The set of computed candidate paths (i.e., the list of up to $K$ explicit route objects - EROs) is provided to RWA PCE within the PCEP PCRep message. The RWA PCE performs RWA on the basis of the network information (including detailed link wavelength availability) stored and dynamically updated in its TED. In our implementation we apply a typical RWA: first-fit WA on the least loaded (validated) path. The selected ERO and wavelength is finally returned to the requesting PCC through a PCEP PCRep message. If no path and wavelength is found, the request is rejected.

The second considered EIV+RWA implementation is derived from the previous IV+RWA. As in IV+RWA, a PCReq message is received by the IV PCE including end-points, bit-rate and candidate objects. Then, differently from the previous case, the PCRep message is extended with a novel optional object, called GB (here used for $100 \mathrm{~Gb} / \mathrm{s}$ requests), to be possibly associated to each one of the returned paths. This object specifies the GB that needs to be accounted, among 100 and $10 \mathrm{~Gb} / \mathrm{s}$ lightpaths, during RWA (if a path is acceptable in the worst-case scenario, GB is 0 and the GB object is omitted, otherwise GB is included). In this way, also paths not guaranteeing acceptable BER with the worst-case approach (but feasible with GB) can be considered for connection set up. The RWA PCE finally selects the least loaded path. To limit the usage of GB, $100 \mathrm{~Gb} / \mathrm{s}$ lightpaths with GB indication are packed on neighbor wavelengths and last-fit WA is applied to those lightpaths, while first-fit to the others.

\section{EXPERIMENTAL DEMONSTRATION}

The two considered IV+RWA and EIV+RWA have been evaluated on the multi bit-rate testbed in Fig. 1 and through emulations on the network topology in [3]. The testbed implements DP-QPSK $100 \mathrm{~Gb} / \mathrm{s}$ signal generation through a tunable external cavity laser (ECL) with a line width of about $100 \mathrm{KHz}$, modulated using an integrated LiNbO3

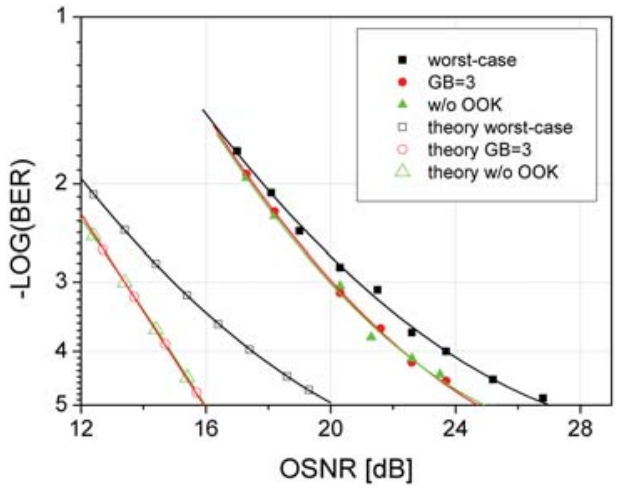

Fig. 2. BER vs. OSNR of the $100 \mathrm{~Gb} / \mathrm{s}$ lightpath.
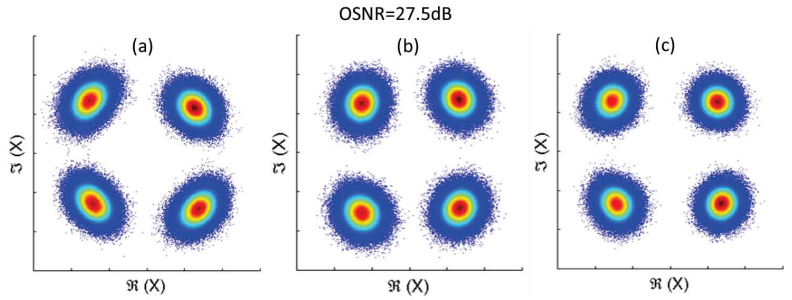

Fig. 3. Symbol constellation of a single $100 \mathrm{~Gb} / \mathrm{s}$ DP-QPSK polarization: (a) in the worst-case scenario; (b) in the absence of OOK channels; (c) in the presence of $\mathrm{OOK}$ channels with $\mathrm{GB}=3$.

double nested Mach Zehender modulator. A $50 \mathrm{~Gb} / \mathrm{s}$ QPSK optical signal is obtained by driving the in-phase (I) and the quadrature $(\mathrm{Q})$ modulator branches with two $25 \mathrm{~Gb} / \mathrm{s}$ pseudo random bit sequences (PRBS) of length $2^{11}-1$ (D1,D2). D1 and D2 are generated with a differential delay of 10 bit periods by properly multiplexing four $12.5 \mathrm{~Gb} / \mathrm{s}$ PRBS. Finally, $100 \mathrm{~Gb} / \mathrm{s}$ DP-QPSK transmission is obtained by emulating polarization multiplexing through a $50 / 50$ beam splitter, a delay line, and a polarization beam combiner (PBC) (Fig. 1). The $10 \mathrm{~Gb} / \mathrm{s}$ OOK signals are generated by four transponders $\left(T_{i}\right)$ at $\lambda_{10, i}(\mathrm{i}=1,2,3,4)$. A $100 \mathrm{GHz}$ spaced array waveguide grating (AWG) is used as multiplexer for the $10 \mathrm{~Gb} / \mathrm{s}$ OOK and $100 \mathrm{~Gb} / \mathrm{s}$ DP-QPSK signals. The link consists of three spans of $80 \mathrm{~km}$ standard single-mode fiber, each one followed by a dispersion compensating fiber. Finally, channels are dropped and received (coherent detection with digital post processing as in [8] is used for the $100 \mathrm{~Gb} / \mathrm{s}$ ). To show the impact of XPM in the testbed, the BER performance of the $100 \mathrm{~Gb} / \mathrm{s}$ lightpath, depicted in Fig. 2, has been evaluated by varying the received OSNR. In a first experiment, the $100 \mathrm{~Gb} / \mathrm{s}$ signal wavelength was set considering the worst-case scenario $\left(\lambda_{100, \text { WORST }}\right.$ in Fig. 1). In a second experiment, $\mathrm{GB}=3$ is considered among the OOK and the DP-QPSK signals $\left(\lambda_{100, \mathrm{~GB}}\right)$. In a third experiment, with $\lambda_{100, \mathrm{WORST}}$, OOK signals were switched off. By comparing the measured BER obtained with $\mathrm{GB}=3$ and OOK switched off, the experimental results confirm that (at least) three channels guarantee negligible XPM on $100 \mathrm{~Gb} / \mathrm{s}$ DP-QPSK due to OOK signals. $8 \mathrm{~dB}$ penalty is the difference between experimental and theoretical performance, which is mainly due to front-end imperfections and narrow filtering from the receiver, while the error floors at high OSNR are due to electronic noise. Finally, XPM in the worst-case scenario corresponds to an OSNR penalty of about $1.5 \mathrm{~dB}$ at a BER 


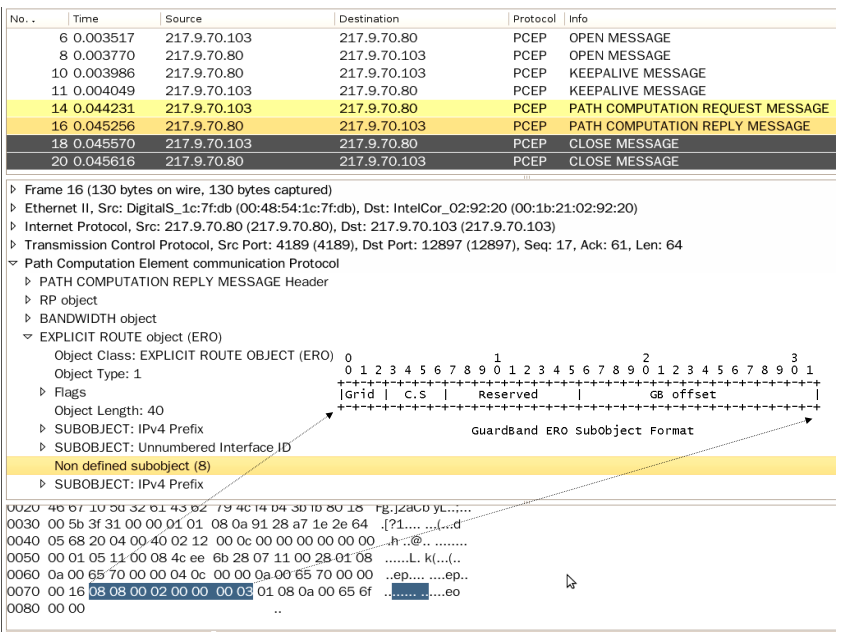

Fig. 4. PCEP capture.

of $10^{-3}$. The effects of XPM on the $100 \mathrm{~Gb} / \mathrm{s}$ signal are also visible in Fig. 3, which shows the received symbol constellation on a single polarization of the $100 \mathrm{~Gb} / \mathrm{s}$ DPQPSK in the presence and in the absence of the OOK signals. For each transmitted symbol, the corresponding distribution of the received samples has a circular symmetry in the absence of OOK channels in Fig. 3(b) or when GB=3 in Fig. 3(c), as expected for an additive white Gaussian noise channel, while it is bent around the origin in Fig. 3(a) due to XPM.

In the experimental demonstration, a $100 \mathrm{~Gb} / \mathrm{s}$ request has been submitted to the PCE. Fig. 4 shows a capture of the PCEP messages exchanged between the IV PCE and the RWA PCE, both implemented in $\mathrm{C}++$, when the PCE implementation is EIV+RWA. The IV PCE applies the IV tools considering also the experimental value of $8 \mathrm{~dB}$ penalty for the QoT estimation of $100 \mathrm{~Gb} / \mathrm{s}$ lightpath. The PCEP session is first established through the initial handshake including Open and Keepalive messages (packets 6-11), then a PCEP Request is sent to the IV PCE (packet 14). In the considered case, the physical link operates at the OSNR value of 20.5 $\mathrm{dB}$ and $\mathrm{BER}_{\mathrm{TH}}=10^{-3}$. The PCE identifies as unacceptable the path in the worst-case scenario but as acceptable with $\mathrm{GB}=3$ (i.e., $3.2 \mathrm{~nm}$ ). The PCEP Reply (packet 16) includes the ERO and the GB object. The IV PCE path computation and validation time is about $200 \mu \mathrm{s}$. Also, a simulated PanEuropean WSON [3] has been enforced within the PCE TED to assess the performance of the proposed PCE implementations in terms of computation time and number of exploited paths. The considered WSON, designed for $10 \mathrm{~Gb} / \mathrm{s}$, includes 17 nodes, 33 bi-directional links, 40 wavelengths, and physical parameters as in the testbed. Considering just the $10 \mathrm{~Gb} / \mathrm{s}$ single bit-rate, XPM effects are negligible, and IV+RWA and EIV+RWA provide the same results. Indeed, they practically operate on the same set of paths (e.g., the set of feasible shortest paths in terms of hops is $\left.P_{s, d},\left|P_{s, d}\right|=496\right)$. Then, in the same WSON, a $10-100 \mathrm{~Gb} / \mathrm{s}$ multi bit-rate scenario is considered. In this case, IV+RWA and EIV+RWA provide different results for $100 \mathrm{~Gb} / \mathrm{s}$ since they consider different sets of paths. Tab. I shows that the set of candidate paths provided by IV+RWA corresponds to the $24 \%$ of $P_{s, d}$ for $100 \mathrm{~Gb} / \mathrm{s}$, while to $37 \%$ by EIV+RWA. Moreover, the exploitation of $\mathrm{GB}=3$ does not negatively impact the average wavelength
TABLE I

PERCENTAGE OF ACCEPTABLE PATHS UTILIZED FOR ROUTING AND UTILIZED WAVELENGTHS PER LINK (@500 ERLANG)

\begin{tabular}{|c|c|c|}
\hline & IV+RWA & EIV+RWA \\
\hline acceptable paths & $24.1 \%$ & $37.5 \%$ \\
\hline utilized wavelengths per link & $25 \%$ & $30 \%$ \\
\hline
\end{tabular}

utilization (shown for 500 Erlang considering Poisson traffic with uniform distribution of source-destination pairs and bitrate requests), which is larger in the case of EIV+RWA with respect to IV+RWA, when $10 \mathrm{~Gb} / \mathrm{s}$ lightpaths are active in the network. A slightly higher number of wavelengths is used with EIV+RWA than with IV+RWA since more paths are exploited with GB. With EIV+RWA, the maximum time experienced to communicate, for a single request, the ERO to the RWA PCE is $3.2 \mathrm{~ms}$, including $2.3 \mathrm{~ms}$ for the EIV computation of $K=6$ candidate paths and the communication time for the PCEP message exchange (one PCReq and one PCRep with 6 EROs, including 2 EROs with the GB object). With IV+RWA on the same node pair, the time experienced to get the ERO at the RWA PCE is $2.7 \mathrm{~ms}$, including $1.9 \mathrm{~ms}$ for the IV computation (one PCReq and one PCRep with just 4 EROs).

\section{CONCLusions}

Two impairment-aware PCE architectures of type IVcandidates+RWA are presented for multi bit-rate $10-100 \mathrm{~Gb} / \mathrm{s}$ WSON, where XPM is particularly a detrimental effect. Experimental results are provided to show their performance in terms of percentage of exploited paths and path delivery time. Computation and communication times result to be extremely fast (up to few milliseconds) for both transmission rates. A novel PCEP extension is also proposed to improve the overall effectiveness in network performance by enabling the usage of a larger set of candidate paths without significantly increasing the delivery time. In the long term, the proposed extension could also be exploited against the XPM induced by newly introduced lightpaths (e.g., at $400 \mathrm{~Gb} / \mathrm{s}$ ) using quadrature amplitude modulation (QAM), which exhibits both intensity and phase modulation.

\section{REFERENCES}

[1] N. Sambo, G. Meloni, F. Paolucci, M. Secondini, L. Potì, F. Cugini, and P. Castoldi, "Impairment-aware PCE in multi bit-rate $10-100 \mathrm{~Gb} / \mathrm{s}$ WSON with experimental demonstration," in Proc. of OFC 2011.

[2] A. Bononi, M. Bertolini, P. Serena, and G. Bellotti, "Cross-phase modulation induced by OOK channels on higher-rate DQPSK and coherent QPSK channels," J. Lightw. Technol., vol. 27, no. 18, Sep. 2009.

[3] N. Sambo, M. Secondini, F. Cugini, G. Bottari, P. Iovanna, F. Cavaliere, and P. Castoldi, "Modeling and distributed provisioning in 10-40-100 $\mathrm{Gb} / \mathrm{s}$ multi-rate wavelength switched optical networks," J. Lightw. Technol., vol. 29, no. 9, May 2011.

[4] Y. Lee, G. Bernstein, D. Li, and G. Martinelli, "A framework for the control of wavelength switched optical networks (WSON) with impairments," draft-ietf-ccamp-wson-impairments-04.txt, Oct. 2010, Internet draft.

[5] M. Suzuki and T. Tsuritani, "Management of GMPLS controlled alloptical network considering physical impairments," in Proc LEOS 2009.

[6] R. Martinez, R. Casellas, R. Munoz, and T. Tsuritani, "Experimental translucent-oriented routing for dynamic lightpath provisioning in GMPLS-enabled wavelength switched optical networks," J. Lightw. Technol., vol. 28, no. 8, 2010.

[7] F. Agraz, et al., "Experimental demonstration of centralized and distributed impairment-aware control plane schemes for dynamic transparent optical networks," in Proc. of OFC/NFOEC 2010.

[8] G. Colavolpe, T. Foggi, E. Forestieri, and G. Prati, "Robust multilevel coherent optical systems with linear processing at the receiver," J. Lightw. Technol., vol. 27, no. 13, 2009. 